\title{
Short-Range Correlations in Nuclear Matter at Finite Temperatures and High Densities *
}

\author{
F. Frömel, H. Lenske, U. Mosel \\ Institut für Theoretische Physik, Universität Giessen, Heinrich-Buff-Ring 16, \\ D-35392 Giessen, Germany
}

\begin{abstract}
The density and temperature dependence of the nucleonic single particle spectral function in symmetric nuclear matter at finite temperatures and densities beyond normal nuclear matter density is investigated in a model emphasizing short-range correlations and phase space aspects. A simple but self-consistent approach based on quantum transport theory is used. In particular we consider the density and temperature regime occuring e.g. during the core collapse in a supernova explosion and the subsequent formation of a neutron star. Mean-field effects are incorporated by a Skyrme type potential.
\end{abstract}

Key words: nuclear matter, many-body theory, nucleon spectral function PACS: 21.65.+f, 24.10.Cn

\section{Introduction}

An interesting but only partially explored problem of nuclear many-body theory are contributions from short-range correlations to the binding properties of nuclear matter and finite nuclei. Of particular interest for various regions of nuclear physics ranging from heavy ion collisions to the physics of neutron star formation is the density and temperature dependence of short-range correlations in nuclear matter. In heavy ion collisions like those planned at the Compressed Baryonic Matter (CBM) facility at GSI densities of several times the nuclear matter saturation density $\rho_{0}=0.16 / \mathrm{fm}^{3}$ are reached. Such conditions are also encountered in astrophysical scenarios. When a neutron star is

\footnotetext{
ऋ Work supported by DFG and GSI.

Email address: Frank.Froemel@theo.physik.uni-giessen.de (F. Frömel).
} 
formed in a supernova explosion temperatures of several tens of $\mathrm{MeV}$ and densities far beyond normal nuclear matter density arise. The neutrinos emitted from the nascent neutron star are an important signal that gives information about the ongoing cooling processes. Nucleon correlations, however, affect directly the neutrino opacity in dense nuclear matter. The neutrino cross sections can be significantly reduced due to the presence of correlations in the medium $[1,2]$. Therefore it is important to take nucleon correlations into account when interpreting the neutrino spectra.

So far there has been no consistent investigation of the properties of the nucleon spectral function that covers the full temperature and density range of those scenarios. In this work we extend the approach to short-range correlations in nuclear matter presented initially in $[3,4]$ to the more general cases of arbitrary temperatures and densities. Using direct relations between correlation functions and collision integrals a simple but fully self-consistent model for calculating the nucleon spectral function in an iterative procedure was constructed in refs. [3,4]. The former calculations, however, have been restricted to zero temperature and normal nuclear matter density. The previously obtained results are in surprisingly good agreement with much more sophisticated and numerically extremely involved, "state-of-the-art" calculations utilizing the full machinery of modern nuclear many-body theory $[5,6]$. In particular, the significant population of high-momentum states in the nucleon momentum distribution - which is interpreted as an overall measure for short-range correlations - is described rather well.

In this paper, we reconsider this model and extend it to finite temperatures and densities beyond the nuclear matter equilibrium point. Different from $[3,4]$ this requires a reliable description of the static mean-field self-energy which in the former work could most conveniently be absorbed into a redefinition of the chemical potential. In the present case an explicit treatment of the density and momentum dependence of the mean-field self-energy is necessary since the chemical potential is temperature and density dependent. Calculations for the equation of state for dense matter and finite temperatures have been made using microscopic Hamiltonians, see e.g. [7,8]. In our model we incorporate the static properties of the mean-field by an empirical, Skyrme-type energy density functional $[9,10,11]$. By this choice thermodynamical consistency is also ensured by fulfilling the Hugenholtz-van Hove theorem [12] at mean-field level. This approach gives rise to a potential that is independent of energy but depends on momentum, temperature, and density. However, because of the Skyrme-typical restriction to terms of at most quadratic order in the single particle momenta the momentum dependence can be re-expressed immediately by an effective mass in the kinetic energy term.

Calculations similar to ours have been done by Alvarez-Ruso et al. [13] for densities from half to twice $\rho_{0}$ and temperatures up to $20 \mathrm{MeV}$. In their "semi- 
phenomenological" approach they have evaluated second order diagrams taking the magnitude of the NN interaction from experiment. However, they did not perform their calculations self-consistently and did not consider long-range contributions from the mean-field. Note that the basis of their work was also a model for cold nuclear matter [14]. Self-consistent approaches to the nucleon spectral function in nuclear matter have been studied theoretically before, e.g. by Alm et al. [15] and in ref. [16]. More recently, calculations using a self-consistent T-matrix approach have been presented by Bożek $[17,18]$. In [17] the temperature dependence of the spectral function was investigated up to $T=20 \mathrm{MeV}$, but the density dependence has so far not been investigated by Bożek.

In this work we go far beyond the temperature and density ranges of [13][18]. To study the role of short-range correlations in supernovae and heavy ion collisions at CBM we consider temperatures up to $70 \mathrm{MeV}$ and densities from $\rho_{0}$ to $3 \rho_{0}$. In section 2 we briefly outline our model. The results of the calculations are presented in section 3 . They are compared to other models (at $T=0$ and finite temperatures) and we discuss the temperature and density dependence of the short-range correlations in nuclear matter. A summary is given in section 4 .

\section{The Model}

The model we present here was introduced in [3,4] for cold $(T=0)$ nuclear matter at saturation density $\rho_{0}$. The underlying Green's function formalism is described in much detail in $[19,20]$. Thus we restrict ourselves here to a short summary of the concept. We show in more detail how to modify the original model $[3,4]$ for the application to arbitrary densities and temperatures. Throughout this paper we consider only symmetric nuclear matter.

On the two-particle-one-hole $(2 p 1 h)$ and one-particle-two-hole $(1 p 2 h)$ level, respectively, the collisional or polarization self-energies $\Sigma^{>}$and $\Sigma^{<}$are given by:

$$
\begin{aligned}
\Sigma^{\gtrless}= & g \int \frac{d^{3} p_{2} d \omega_{2}}{(2 \pi)^{4}} \frac{d^{3} p_{3} d \omega_{3}}{(2 \pi)^{4}} \frac{d^{3} p_{4} d \omega_{4}}{(2 \pi)^{4}}(2 \pi)^{4} \delta^{4}\left(p+p_{2}-p_{3}-p_{4}\right) \overline{\left|\mathcal{M}^{2}\right|} \\
& \times g^{\lessgtr}\left(\omega_{2}, p_{2}\right) g^{\gtrless}\left(\omega_{3}, p_{3}\right) g^{\gtrless}\left(\omega_{4}, p_{4}\right),
\end{aligned}
$$

where $g=4$ is the nucleonic spin-isospin degeneracy factor and $\overline{|\mathcal{M}|^{2}}$ is the square of the nucleon-nucleon scattering amplitude averaged over spin and isospin of the incoming and summed over spin and isospin of the outgoing nucleons. We leave the structure of $\mathcal{M}$ open for the moment and come back 
to it later. The correlation functions $g^{>}$and $g^{<}$in eq.(1) can be rewritten in terms of the spectral function $a(\omega, p)$,

$$
\begin{aligned}
& g^{<}(\omega, p)=i a(\omega, p) f(\omega, p) \\
& g^{>}(\omega, p)=-i a(\omega, p)(1-f(\omega, p))
\end{aligned}
$$

with the phase space distribution function $f(\omega, p)$. In thermal equilibrium $f$ is just the Fermi distribution function depending on the energy and, through the chemical potential $\omega_{F}$, on the density of the system,

$$
f(\omega)=\frac{1}{1+e^{\left(\omega-\omega_{F}\right) / T}}
$$

The previous calculations were greatly simplified by the fact that at zero temperature the Fermi distributions reduce to step functions allowing for considerable simplifications of eq.(1) by taking advantage of closed form analytic expressions $[3,4]$.

The single particle spectral function in (2) is explicitly given as

$$
a(\omega, p)=\frac{\Gamma(\omega, p)}{\left(\omega-\frac{p^{2}}{2 m}-\Sigma^{\mathrm{mf}}-\operatorname{Re} \Sigma^{\mathrm{ret}}(\omega, p)\right)^{2}+\frac{1}{4} \Gamma^{2}(\omega, p)},
$$

where $\operatorname{Re} \Sigma^{\text {ret }}$ is the real part of the collisional self-energy and $\Gamma$ is the width of the spectral function. $\Gamma$ is directly related to the imaginary part of the retarded self-energy,

$$
\Gamma(\omega, p)=-2 \operatorname{Im} \Sigma^{\mathrm{ret}}(\omega, p)=i\left(\Sigma^{>}(\omega, p)-\Sigma^{<}(\omega, p)\right) .
$$

Note, that eq.(4) also includes the mean-field self-energy $\Sigma^{\mathrm{mf}}=\Sigma^{\mathrm{mf}}(p, \rho, T)$ which is not contained in $\Sigma \gtrless$. The purely hermitian $\Sigma^{\mathrm{mf}}$ contains the contributions to the self-energy arising from ground state tadpole diagrams describing mainly long-range correlations.

We can rewrite the first term in the denominator of eq.(4) by expanding $\Sigma^{\mathrm{mf}}(p, \rho, T) \approx \Sigma^{\mathrm{mf}}\left(k_{F}, \rho, T\right)+\left(p^{2}-k_{F}^{2}\right) \Sigma^{\mathrm{mf}^{\prime}}\left(k_{F}, \rho, T\right)+\ldots$ up to $\mathcal{O}\left(p^{2}\right)$. The momentum dependent part of $\Sigma^{\mathrm{mf}}$ can then be absorbed into the kinetic energy term by introducing a density and temperature dependent effective mass $\frac{1}{m^{*}}=\frac{1}{m}\left(1+2 m \Sigma^{\mathrm{mf}^{\prime}}\right)$, as successfully used, e.g., in Skyrme density functionals $[9,10,11]$. We get:

$$
\frac{p^{2}}{2 m}+\Sigma^{\mathrm{mf}}(p, \rho)+\operatorname{Re} \Sigma^{\mathrm{ret}}(\omega, p) \cong \frac{p^{2}}{2 m^{*}}+U_{\mathrm{eff}}(\rho)+\operatorname{Re} \Sigma^{\mathrm{ret}}(\omega, p)
$$


where the effective potential $U_{\text {eff }}$ is the momentum independent part of $\Sigma^{\mathrm{mf}}$. We will discuss the role of $U_{\text {eff }}$ and $\operatorname{Re} \Sigma^{\text {ret }}$ in more detail below.

The equations (1)-(5) describe a self-consistency problem. Typically, such problems cannot be solved directly but iterative procedures are the appropriate methods of solution. Hence, the spectral function is obtained iteratively by the following procedure: Starting with an initial guess for $a(\omega, p)$ in the integrals of eq.(1) an approximation for the width $\Gamma$ is found. Inserting this $\Gamma(\omega, p)$ into eq. (4) yields an improved expression for the spectral function that is inserted into (1) again. This procedure can be repeated until convergence is achieved. In the language of Feynman diagrams the solution of this selfconsistency problem corresponds to the non-perturbative summation over a whole class of diagrams by reinserting the full in-medium Green's functions into the self-energies.

As we have seen above there are two real contributions to the self-energy. One is the mean-field self-energy that is purely real and does not affect the width directly. The other contribution is the real part of the collisional self-energy $\operatorname{Re} \Sigma^{\text {ret }}$. It is related to the imaginary part of the polarization self-energy (i.e., the width of the single particle spectral function) by a dispersion relation, e.g. discussed in [4]. There, the importance of the dispersive contributions was investigated by performing calculations with and without $\operatorname{Re} \Sigma^{\text {ret }}$. Although analyticity is not conserved when $\operatorname{Re} \Sigma^{\text {ret }}$ is neglected [3] it was found that overall the results on spectral functions did not change very much. Taking advantage of this finding we will ignore the dispersive part for the present investigations, thus simplifying the calculations considerably. For a full treatment of $\operatorname{Re} \Sigma^{\text {ret }}$ we refer to $[3,4]$.

More important for our present purpose of studying the density and temperature dependence of short-range correlations are the mean-field potential $U_{\text {eff }}=U_{\text {eff }}(\rho, T)$ and the effective mass $m^{*}=m^{*}(\rho)$. The net effect of the potential $U_{\text {eff }}$ is a temperature and density dependent energy shift of the pole structure of the single particle propagators and hence the spectral functions. For the investigations in $[3,4]$ at $T=0$ and $\rho=\rho_{0}$ such a shift could be absorbed into a re-definition of the chemical potential. This is no longer possible when we look for the density and temperature dependence of our results to which long-range correlations contribute.

The temperature and density dependence of the single-particle potential in nuclear matter has been studied before microscopically, ranging from selfconsistent Brueckner and Brueckner Hartree-Fock approaches e.g. in $[15,16]$ to the variational calculations with 2- and 3-body forces by the Urbana group $[7,8]$. In a different context, the density and momentum dependence of the variational mean-field was studied in [7]. A re-analysis of these rather involved mean-field results by a second order polynomial in $k^{2}-k_{F}^{2}$ leads to an almost 
perfect description of the momentum dependence with an effective mass of $m^{*} / m=0.69$. This confirms the effective mass approach utilized in eq.(6). These findings also confirm the use of a schematic description of the meanfield part, in particular so because the static parts of the self-energies are cancelled to a large extent in dynamical quantities like propagators. Hence, we decide to describe the mean-field in a Skyrme model $[9,10,11]$ for the sake of a most transparent and simple approach.

From the Skyrme energy density functional in standard notation $[9,10,11] U_{\text {eff }}$ and $m^{*}$ in infinite symmetric nuclear matter are obtained by

$$
\begin{aligned}
U_{\text {eff }}(\rho) & =\frac{3}{4} t_{0} \rho+\frac{1}{16}\left(3 t_{1}+5 t_{2}\right) \tau+\frac{3}{16} t_{3} \rho^{2}, \\
\frac{1}{2 m^{*}} & =\frac{1}{2 m}+\frac{1}{16}\left(3 t_{1}+5 t_{2}\right) \rho,
\end{aligned}
$$

where $t_{0}, t_{1}, t_{2}$, and $t_{3}$ are parameters of the Skyrme interaction $[9,10,11]$. The parameter set we have used here is given in Table 1. $\tau$ is the (quasi-particle) kinetic energy density,

$$
\tau=\frac{2}{\pi^{2}} \int_{0}^{\infty} d p \frac{p^{4}}{e^{\left(p^{2}-k_{F}^{2}\right) / 2 m^{*} T}+1}
$$

with $k_{F}^{2}=2 m^{*}\left(\omega_{F}-U_{\text {eff }}\right)$.

It is well known that nuclear matter ceases to be bound beyond a critical density $\rho_{\mathrm{c}}(T)$ where the repulsive kinetic energy term overrules the nuclear attraction. For processes inside a supernova this is, however, not a crucial problem. The core collapse is not driven by nuclear, but by gravitational forces. Eventually, the repulsive nuclear potential even stops the collapse when several times nuclear matter density is reached.

For a complete definition of the approach we also have to specify the scattering amplitude $\mathcal{M}$ in the collision integrals (1). Taking into account the observation of Lehr et al. $[3,4]$ that the short-range correlations primarily depend on global properties of the interactions on ranges of the (repulsive) vector meson masses and the available phase space we will also use a constant matrix element in our calculations, corresponding to an effective zero-range contact interaction.

The value of $\mathcal{M}$ will be chosen independent of temperature and density of the system. It is noteworthy that until now a description of short-range correlations including a fully self-consistent description of the interactions is still pending, although first attempts in that direction have been made. In such calculations, e.g. by the Rostock group [15] or, more recently, by Bozek $[17,18]$ 
significant variations of the interactions with temperature are not obtained. Accepting that short-range correlations are due to short-range interactions, a strong dependence of the interaction matrix element is not very likely, either.

In order to enforce the convergence of integrations involving $\mathcal{M}$ and extending over the space of $2 p 1 h$ and $1 p 2 h$ configurations, respectively, we introduce a form factor such that the collision integrals in eq.(1) are cut off at large $\omega$. Lehr et al. had introduced a form factor that acts on the total energies and momenta of the collisions corresponding to eq.(1). This form factor did only affect the results at $\omega>\omega_{F}$. For the occupied states below $\omega_{F}$ the results remained unchanged. Due to the density dependence of the chemical potential, however, this form factor is not a sensible choice for our calculations.

Different to the approach in $[3,4]$ we use here a form factor acting on the tchannel and suppressing processes with large energy and momentum transfers (see [21] for details):

$$
F\left(\omega_{\mathrm{t}}, \vec{p}_{\mathrm{t}}\right)=\frac{\Lambda^{4}}{\Lambda^{4}+\left(m_{\mu}^{2}-\omega_{\mathrm{t}}^{2}+p_{\mathrm{t}}^{2}\right)^{2}},
$$

where $\omega_{\mathrm{t}}=\omega-\omega_{3}$ and $\vec{p}_{\mathrm{t}}=\vec{p}-\vec{p}_{3}$. The mass $m_{\mu} \sim \mathcal{O}\left(m_{\rho}\right)$ corresponds to the typical mass of an exchanged meson in nucleon scattering processes and $\Lambda$ defines the scale of the cutoff. Note that $F$ has no pole on the real axis.

\section{Numerical Details and Results}

We have calculated the nucleon spectral function in nuclear matter at temperatures from 10 to $70 \mathrm{MeV}$ and at densities $\rho=\rho_{0} \cdots 3 \rho_{0}\left(\rho_{0}=0.16 \mathrm{fm}^{-3}\right)$. In the calculations we used the Skyrme force type SIII' to generate a mean-field potential and an effective mass $m^{*}$ (cf. $\left.[9,10,11]\right)$. The corresponding set of parameters is given in Table 1. In infinite nuclear matter at zero temperature one finds for this choice of parameters a binding energy of $-16.1 \mathrm{MeV}$ and a mass ratio of $m^{*} / m=0.76$ at a Fermi momentum of $k_{F}=1.29 \mathrm{fm}^{-1}\left(\rho=\rho_{0}\right)$. For the mean-field potential a value of $U_{\text {eff }}=-61.6 \mathrm{MeV}$ is found. Compared to the variational results of Wiringa [7] the saturation properties are slightly different with an equilibrium point shifted to a somewhat lower density. The larger effective mass indicates a weaker momentum dependence of the SIII' interaction.

The averaged scattering amplitude was adjusted to the value ${ }^{1}\left(\overline{|\mathcal{M}|^{2}}\right)^{1 / 2}=$

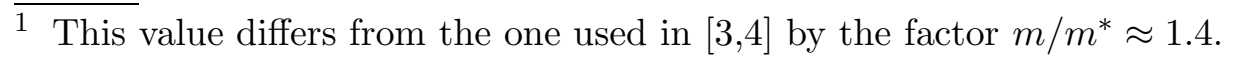


$309 \mathrm{MeV} \mathrm{fm}^{3}$ so that the results of the many-body calculations of Benhar et al. for $T=0$ [5] are reproduced. The cutoff parameter $\Lambda$ of the form factor (10) was set to $1.2 \mathrm{GeV}$ and the mass $m_{\mu}$ to $600 \mathrm{MeV}$. To verify that this is a reasonable choice for the parameters we compare the on-shell width $\Gamma_{\text {on }}(\omega)=$ $\Gamma(\omega, p(\omega))$, where $p(\omega)=\left(2 m^{*}\left(\omega-U_{\text {eff }}\right)\right)^{1 / 2}$, from our calculation to the results of Benhar et al. [5] and Baldo et al. [6]. In Fig. 1 the on-shell widths are shown for normal nuclear matter density and $T=0$. As it can be seen, our result is in good agreement with the sophisticated many-body calculation of Benhar et al. and supports the choice of the parameters. Note that the discrepancies between the results of Baldo and Benhar are explained in [5] by the different NN interactions that are employed.

In the following we will show the results of our calculations and investigate the density and temperature dependence of the short-range correlations. First, we present cuts of the nucleon spectral function at a constant momentum to illustrate the general role of temperature and density. Fig. 2 shows the spectral function for temperatures of 0,10 and $50 \mathrm{MeV}$ at normal nuclear matter density. The effect of higher temperatures is clearly visible. The gap at the Fermi energy gets smeared out for $T=10 \mathrm{MeV}$ and has almost vanished at $T=50 \mathrm{MeV}$. This phenomenon is directly related to the fact that for non-vanishing $\mathrm{T}$ - in contrast to a system at zero temperature - states at energies above $\omega_{F}$ are populated. The states at the Fermi surface cease to be quasi-stable.

In Fig. 3 the spectral function is shown for three different densities at a constant temperature of $10 \mathrm{MeV}$. Since the chemical potential depends on the density the three curves are shifted along the energy axis. Note that the relation $\mu \sim \rho^{1 / 3}$ for the free Fermi gas does not hold here as it can be directly seen for $T=0$, where $\mu=\tau+\operatorname{Re}\left(\Sigma\left(k_{F}\right)\right)$. One should not get mislead by the observation that the on-shell peaks at lower densities are broader than the peak at the highest density. This effect is due to the fact that the on-shell energy lies closer to the Fermi energy for the highest density. This induces a stronger suppression of the on-shell peak than in the other cases.

Figs. 2 and 3 give a first impression of the spectral functions. To investigate the temperature and density dependence of the short-range correlations, however, we use the better suited on-shell width of the spectral function, $\Gamma_{\text {on }}(\omega)$. In Fig. 4 we show our results for $\Gamma_{\text {on }}(\omega)$ at temperatures of 10 and $20 \mathrm{MeV}$ and densities of $\rho_{0}$ and $2 \rho_{0}$. Also shown are the results of Alvarez-Ruso et al. [13]. As we have already seen in Figs. 2 and 3 the width of the states at the Fermi surface does not drop to zero for finite temperatures. Increasing the temperature leads to larger values of $\Gamma_{\text {on }}$ in the vicinity of the Fermi surface while the size and the shape of $\Gamma_{\text {on }}$ do not change significantly at higher energies. Increasing the density shows the opposite effect. $\Gamma_{\text {on }}$ is left unchanged in the vicinity of the Fermi surface while the slope at high energies 
increases. Compared to [13] our results seem to be shifted by approximately $5 \mathrm{MeV}$ to higher values of $\Gamma_{\text {on }}$. The shift can be explained by the differences in the NN interactions that were used in both calculations. The shape of the curves is in good agreement for $\omega-\omega_{F}<150 \mathrm{MeV}$. At higher energies the form factor that was used in our calculations affects the shape of $\Gamma_{\text {on }}$, e.g. leading to the observed curvature in our results.

Qualitatively our results (for $\rho=\rho_{0}$ and $T \leq 20 \mathrm{MeV}$ ) are similar to those obtained in the self-consistent T-matrix approach by Bożek $[17,18]$ but on a quantitative level significant differences are found. However, the deviations range within the uncertainties introduced using different nucleon-nucleon interactions in fully microscopic calculations. This is evident by comparing the results in [18], based on the Paris NN potential, e.g. to the variational results of Benhar et al. [5], obtained with the Argonne NN potential. The reason for these deviations is found in the fact that by the way of deriving these empirical interactions from NN data they are guaranteed to agree in their predictions of on-shell quantities, as e.g. free space NN scattering phase shifts, but might differ in the off-shell region. While we work with a constant coupling in combination with a t-channel form factor Bożek tends to use strongly momentum dependent interactions like the Paris potential (see [18] for details). Similar observations are discussed in [18] when results of different approaches - including [3] and [17] - are compared. Note that the same effect has already shown up in Fig. 1 for the results of Benhar [5] and Baldo [6].

The temperature dependence of the on-shell width at various densities is illustrated in Fig. 5. The effect of filling the gap at the Fermi surface with increasing temperature is clearly visible. At all densities one observes a levelling off in the energy dependence of $\Gamma_{\text {on }}(\omega)$ at the highest temperatures indicating the gradual changes in the available phase space in a heated system.

The behavior seen in Fig. 5 deserves a closer inspection. Before doing so we should take into account that the chemical potential $\omega_{F}$ depends strongly on the nucleon density, as was shown in Fig. 3. Thus one has to be careful at which energy $\omega$ and momentum $p(\omega)$, respectively, the widths for different temperatures and densities are compared. This problem is avoided by averaging the on-shell width over the occupied states and compare this $\left\langle\Gamma_{\text {on }}\right\rangle$ for the different $\rho$ and T. Fig. 6 shows the overall behavior of $\left\langle\Gamma_{\text {on }}\right\rangle$ for the full temperature and density range that was covered in our calculations. Note that one gets a very similar picture when $\Gamma_{\text {on }}(\langle\omega\rangle)$, i.e. the on-shell width at the averaged energy of the occupied states, is displayed instead. The general features of this figure are thus universal. It can be seen in Fig. 6 that the on-shell width ranges from $10 \mathrm{MeV}$ at low temperatures up to $40 \mathrm{MeV}$ at high temperatures. $\left\langle\Gamma_{\text {on }}\right\rangle$ rises almost linearly with the temperature at medium and high densities. At normal nuclear matter density the dependence is only linear for temperatures below $\approx 45 \mathrm{MeV}$ and gets weaker at higher temperatures. The density 
dependence of $\left\langle\Gamma_{\text {on }}\right\rangle$ that can be derived from Fig. 6 has a more complicated structure. As long as the density is low $\left(\rho<2 \rho_{0}\right)\left\langle\Gamma_{\text {on }}\right\rangle$ does increase with the density just as one would expect naively. At densities above $2 \rho_{0}$, however, the density dependence becomes very weak - in particular for temperatures above $30-40 \mathrm{MeV}$. Since we take the on-shell width as a measure for short-range correlations this means that the correlations saturate at densities above two times normal nuclear matter density.

The explanation for the saturation is rather simple. Pauli-blocking suppresses nucleon collisions (the source of the correlations) with final states below the Fermi surface. Since the chemical potential depends strongly on the nucleon density more (kinematically allowed) final states are blocked at higher $\rho$. At high $\rho$ there are also more collision partners (initial states) for a particle with given energy and momentum but this cannot compensate the suppression from Pauli-blocking. To understand this one has to look at the total collision rate for a nucleon with given $\omega$ and $p$ with the nucleons of the medium (cf. eq.(1)). There are two final states that are affected by Pauli-blocking but there is only one initial state from below the Fermi surface. Thus the effect of increased Pauli-blocking is more important than the gain of initial states.

To verify that this explanation is correct we have made additional calculations in which Pauli-blocking was switched off. The result is shown in Fig. 7. It can be clearly seen that without Pauli-blocking there is a linear dependence of $\left\langle\Gamma_{\text {on }}\right\rangle$ on both, temperature and density, over the full range covered by our calculations. This is exactly the picture one would naively expect when Pauliblocking is not taken into account. Thus Pauli-blocking is in fact the correct explanation for the saturation of the short-range correlations at high densities.

\section{Summary and Conclusions}

In this paper we have presented an approach to short-range correlations in nuclear matter at finite temperatures and high densities. Based on the work of Lehr et al. [3,4] we have constructed a simple but self-consistent model that goes beyond the quasi-particle approximation. The interactions between the nucleons were described by a constant matrix element in combination with a form factor that limits the energy and momentum transfer in the collisions. In $[3,4]$ the averaged coupling constant has led to excellent results for a system at zero temperature. We have not calculated the mean-field contributions selfconsistently within the model. Instead, a Skyrme-type interaction model was incorporated to assure a realistic thermodynamical behavior of the chemical potential.

We have calculated the spectral function of nucleons in symmetric nuclear 
matter at temperatures from 10 to $70 \mathrm{MeV}$ and densities from $\rho_{0}$ to $3 \rho_{0}$. This has been the first time that this large regime is investigated consistently within the same model. It was shown that our results are in good agreement with other calculations at temperatures up to $20 \mathrm{MeV}$ and densities up to $2 \rho_{0}$. The width of the spectral function was used to get informations about the temperature and energy dependence of the short-range correlations. We have found that the correlations scale approximately linear with temperature. At high densities, however, the correlations saturate, an effect that can be explained by Pauli-blocking.

The model we have presented here is open for further improvements. Using separate spectral functions for protons and neutrons and introducing an isospin dependent coupling it would also be possible to investigate isospin asymmetric nuclear matter.

\section{Acknowledgments}

We thank Stefan Leupold and Jürgen Lehr for helpful discussions during the preparation of this work. In addition we would like to thank J. Lehr for providing the original computer code that was used in [3]. 


\section{References}

[1] R. F. Sawyer, Phys. Rev. Lett. 75 (1995) 2260.

[2] S. Reddy, J. Pons, M. Prakash, J.M. Lattimer, astro-ph/9802312.

[3] J. Lehr, M. Effenberger, H. Lenske, S. Leupold, U. Mosel, Phys. Lett. B483 (2000) 324.

[4] J. Lehr, H. Lenske, S. Leupold, U. Mosel, Nucl. Phys. A703 (2002) 393.

[5] O. Benhar, A. Fabrocini, S. Fantoni, Nucl. Phys. A505 (1989) 267; O. Benhar, A. Fabrocini, S. Fantoni, Nucl. Phys. A550 (1992) 201.

[6] M. Baldo, I. Bombaci, G. Giansiracusa, U. Lombardo, C. Mahaux, R. Sartor, Nucl. Phys. A545 (1992) 741.

[7] R. B. Wiringa, Phys. Rev. C38 (1988) 2967.

[8] B. Friedman, V. R. Pandharipande, Nucl. Phys. A361 (1981) 592.

[9] G. Sauer, H. Chandra, U. Mosel, Nucl. Phys. A264 (1976) 221.

[10] M. Beiner, H. Flocard, N. van Giai, P. Quentin, Nucl. Phys. A238 (1975) 29.

[11] D. Vautherin, D. M. Brink, Phys. Rev. C5 (1972) 626.

[12] N.M. Hugenholtz, L. van Hove, Physica 24363 (1958).

[13] L. Alvarez-Ruso, P. Fernandez de Cordoba, E. Oset, Nucl. Phys. A606 (1996) 407.

[14] P. Fernandez de Cordoba, E. Oset, Phys. Rev. C46 (1992) 1697.

[15] T. Alm, G. Röpke, A. Schnell, N. H. Wong, H. S. Köhler, Phys. Rev. C53 (1996) 2181.

[16] F. de Jong, H. Lenske, Phys. Rev. C56 (1997) 154.

[17] P. Bożek, Phys. Rev. C59 (1999) 2616.

[18] P. Bożek, Phys. Rev. C65 (2002) 054306.

[19] L. P. Kadanoff, G. Baym, Quantum Statistical Mechanics, Benjamin, New York (1962).

[20] P. Danielewicz, Ann. Phys. 152 (1984) 305.

[21] T. Feuster, U. Mosel, Phys. Rev. C58 (1998) 457. 


\begin{tabular}{|c|c|c|c|c|c|}
\hline $\begin{array}{c}t_{0} \\
\left(\mathrm{MeV} \cdot \mathrm{fm}^{3}\right)\end{array}$ & $\begin{array}{c}t_{1} \\
\left(\mathrm{MeV} \cdot \mathrm{fm}^{5}\right)\end{array}$ & $\begin{array}{c}t_{2} \\
\left(\mathrm{MeV} \cdot \mathrm{fm}^{5}\right)\end{array}$ & $\begin{array}{c}t_{3} \\
\left(\mathrm{MeV} \cdot \mathrm{fm}^{6}\right)\end{array}$ & $\begin{array}{c}x_{0} \\
\left(\mathrm{MeV} \cdot \mathrm{fm}^{5}\right)\end{array}$ \\
\hline \hline-1133.4 & 395 & -95 & 14000 & 0.49 & 120 \\
\hline
\end{tabular}

Table 1

The parameters of the Skyrme force SIII' [9] used in the calculations. For completeness this table also contains the spin-interaction parameter $x_{0}$ and the spin-orbit parameter $W_{0}$.

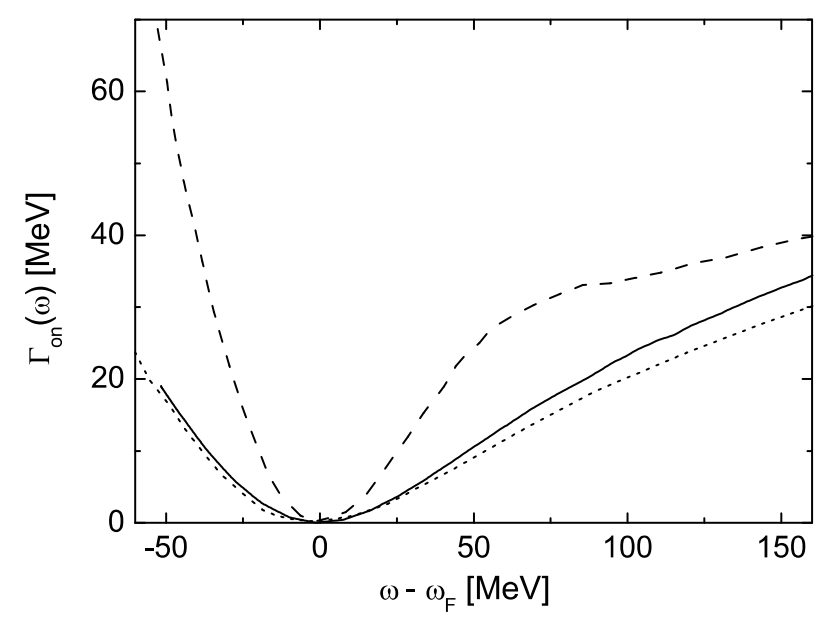

Fig. 1. The on-shell width $\Gamma_{\text {on }}(\omega)$ as a function of $\omega-\omega_{F}$ for $T=0$ and normal nuclear matter density. The solid line shows the result of our calculation, the dashed line the result of Benhar et al. [5], and the dotted line the result of Baldo et al. [6].

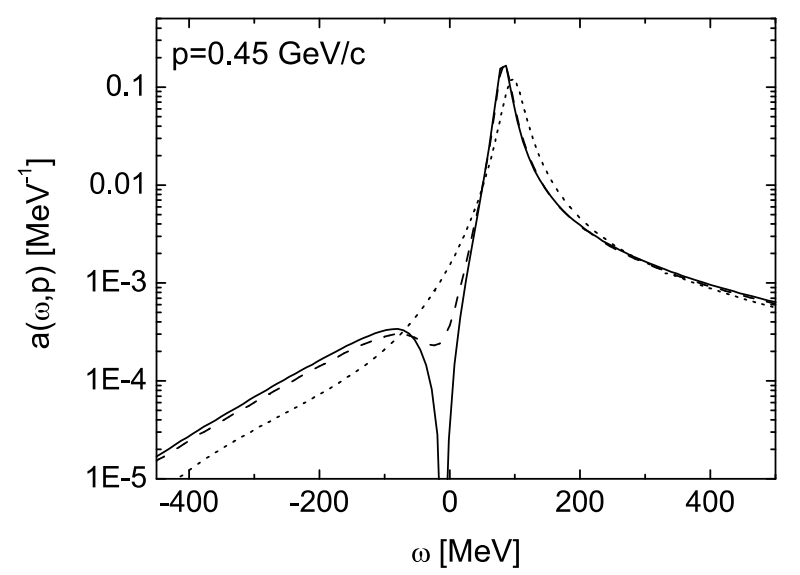

Fig. 2. The nucleon spectral function at $T=0 \mathrm{MeV}$ (solid line), $T=10 \mathrm{MeV}$ (dashed line) and $T=50 \mathrm{MeV}$ (dotted line) for normal nuclear matter density. All cuts were made at a constant momentum of $p=0.45 \mathrm{GeV}$. 


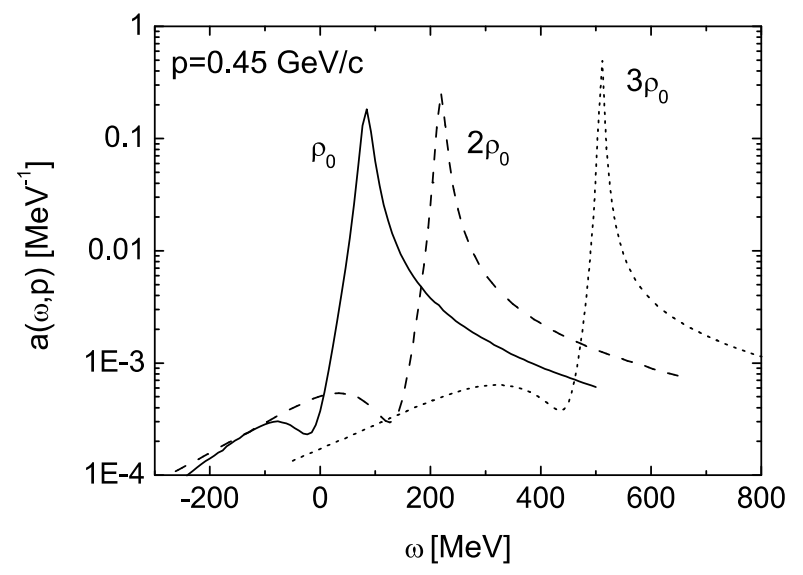

Fig. 3. The nucleon spectral function for normal nuclear matter density (solid line), a two times higher density (dashed line), and a three times higher density (dotted line) at $T=10 \mathrm{MeV}$. All cuts were made at a constant momentum of $p=0.45 \mathrm{GeV}$.

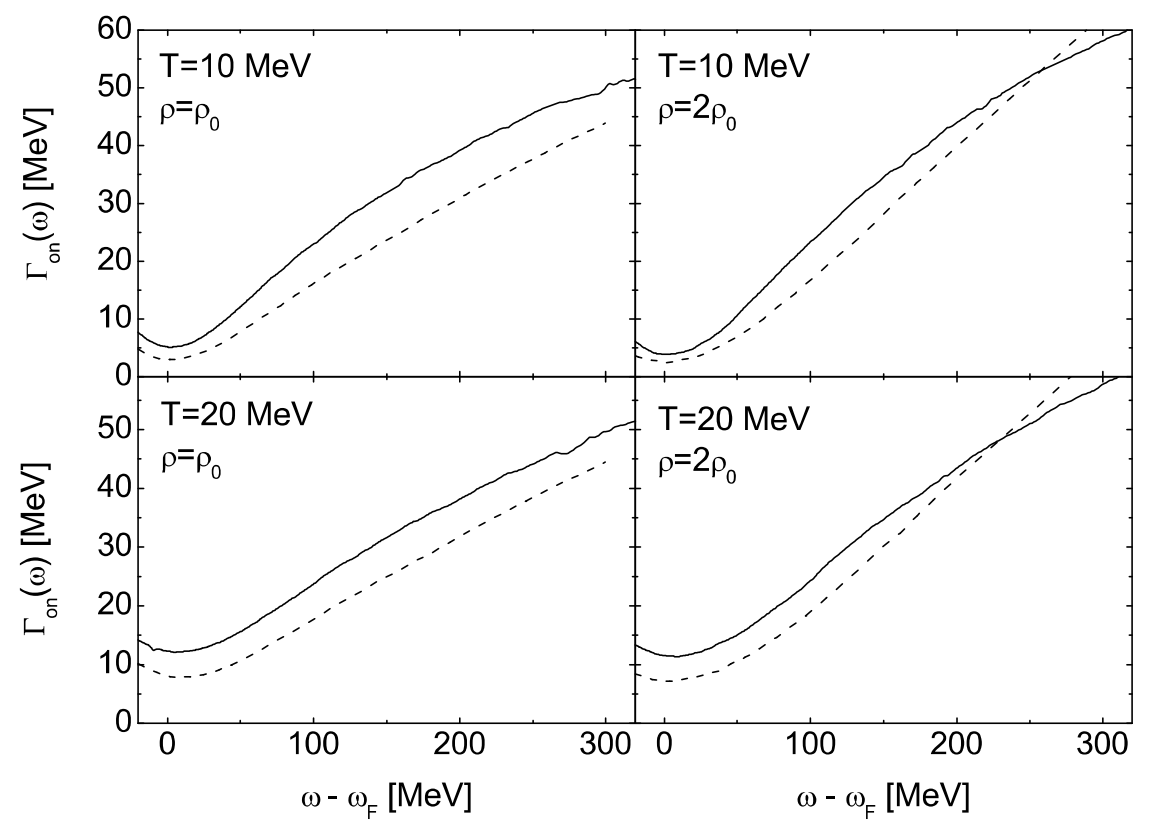

Fig. 4. The on-shell width $\Gamma_{\text {on }}(\omega)$ as a function of $\omega-\omega_{F}$ for two temperatures and densities. The solid lines show the results of our calculations, the dashed lines show the results of Alvarez-Ruso et al. [13]. 


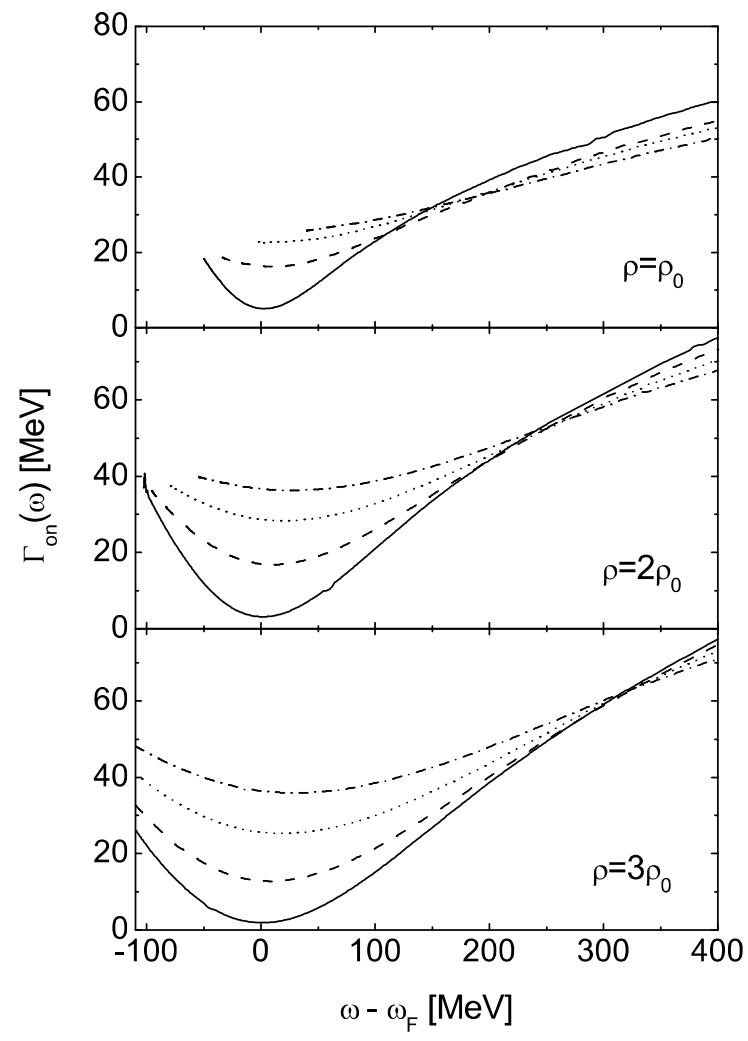

Fig. 5. The on-shell width $\Gamma_{\text {on }}(\omega)$ as a function of $\omega-\omega_{F}$ for different temperatures and densities. Results for $T=10,30,50,70 \mathrm{MeV}$ are indicated by solid, dashed, dotted and dashed-dotted lines, respectively.

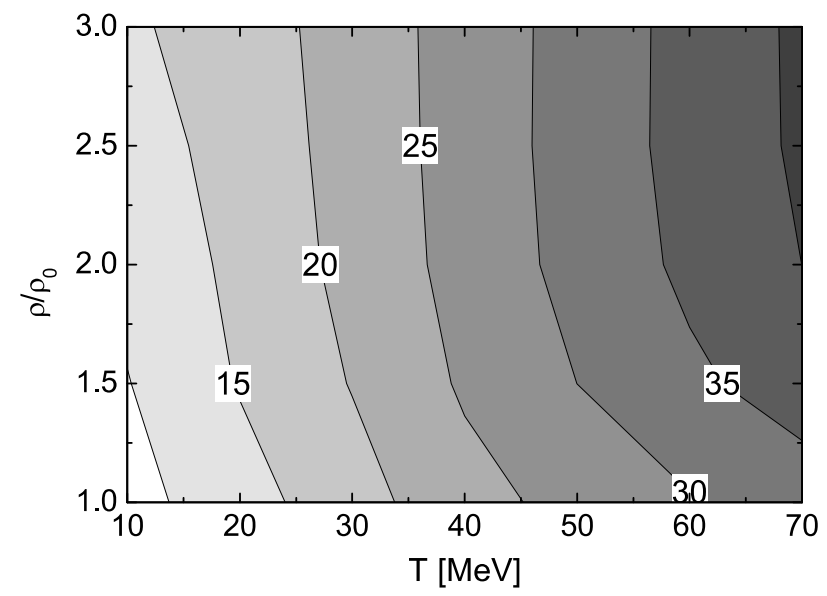

Fig. 6. The on-shell width $\left\langle\Gamma_{\text {on }}\right\rangle$ in the $(T, \rho)$-plane $(\Gamma$ in $\mathrm{MeV})$. For each temperature and density $\Gamma_{\text {on }}$ has been averaged over the occupied states. 


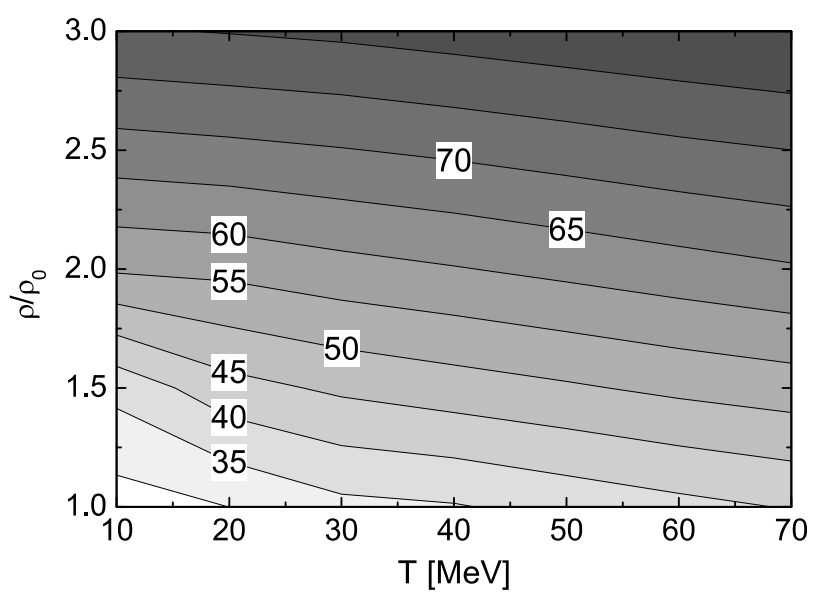

Fig. 7. The on-shell width $\left\langle\Gamma_{\text {on }}\right\rangle$ in the $(T, \rho)$-plane $(\Gamma$ in $\mathrm{MeV})$ when Pauli-blocking is switched off in the calculations. For each temperature and density $\Gamma_{\text {on }}$ has been averaged over the occupied states. 